Proceedings of the 2012 Winter Simulation Conference

C. Laroque, J. Himmelspach, R. Pasupathy, O. Rose, and A. M. Uhrmacher, eds.

\title{
AN EFFICIENT SIMULATION-BASED OPTIMIZATION ALGORITHM FOR LARGE-SCALE TRANSPORTATION PROBLEMS
}

\author{
Carolina Osorio \\ Linsen Chong \\ Massachusetts Institute of Technology \\ 77 Massachusetts Ave, Cambridge, MA 02139, USA
}

\begin{abstract}
This paper applies a computationally efficient simulation-based optimization (SO) algorithm suitable for large-scale transportation problems. The algorithm is based on a metamodel approach. The metamodel combines information from a high-resolution yet inefficient microscopic urban traffic simulator with information from a scalable and tractable analytical macroscopic traffic model. We then embed the model within a derivative-free trust region algorithm. We evaluate its performance considering tight computational budgets.

We illustrate the efficiency of this algorithm by addressing an urban traffic signal control problem for the full city of Lausanne, Switzerland. The problem consists of a nonlinear objective function with nonlinear constraints. The problem addressed is considered large-scale and complex both in the fields of derivative-free optimization and simulation-based optimization. We compare the performance of the method to a traditional metamodel method.
\end{abstract}

\section{INTRODUCTION}

\subsection{Motivation}

In the field of urban transportation, detailed traffic simulators have been used to provide insights in the design and operations of underlying networks. This work is motivated by the use of highly detailed traffic simulators (known as microscopic simulators) that describe the behavior of individual travelers as well as the technologies of individual vehicles. Microscopic simulators model how each traveler makes decisions such as the choice of travel mode, departure-time, travel route, or how they react to real-time traffic information. These descriptions are based on the use of disaggregate behavioral models. Additionally, these simulators can account for the vehicle-specific technologies and provide a detailed description of how these technologies perform in congested urban settings.

Nevertheless, since numerous stochastic models are embedded in these simulators, they yield stochastic nonlinear outputs that are computationally costly to evaluate. Thus, their use is mainly limited to what-if analysis, i.e., experts use them to evaluate a set of predetermined strategies (e.g., novel network designs or traffic management schemes). This work considers the development of a simulation-based optimization (SO) framework that enables the use of these simulators in order to derive suitable strategies.

The focus of this work is to develop computationally efficient frameworks such that they can be used by practitioners to address challenging transportation problems (e.g., high-dimensional nonconvex constrained stochastic problems). Our objective is to develop an SO algorithm with good short-term performance, i.e., one that can identify solutions within a limited computational budget. The building block of this paper is an existing SO method that has proven to be efficient to address traffic management problems. This paper uses a scalable formulation of this framework suitable to address large-scale SO problems (Osorio and Bierlaire 2010). This initial method has been extensively and successfully used to address urban transportation problems (Osorio and Bidkhori 2012; Chen, Osorio, and Santos 2012; Osorio and Nanduri 2012). 


\section{Osorio and Chong}

$$
\min _{x, y \in \Omega} E[f(x, y ; p)]
$$

The objective function is the expected value of a given stochastic performance measure. It is a function of a set of continuous decision variables $x$, endogenous variables $y$ and exogenous parameters $p$. In this paper, we illustrate our SO method with a traffic signal control problem, where $f$ is the travel time, $x$ are the green time durations of the phases at different intersections, $y$ includes, for instance, signalized lane capacities, and $p$ includes, for instance, the network topology, the total demand, lane attributes. The feasible space $\Omega$ consists of a set of general, typically nonconvex and differentiable deterministic constraints.

\subsection{Metamodel Methods}

SO methods can be classified as (1) direct-search methods, which rely only on objective function and do not resort to any direct or indirect model building (for reviews see Conn and Scheinberg (2009) and Kolda, Lewis, and Torczon (2003)), (2) direct gradient, which estimate the gradient of the simulation response and (3) metamodel methods, which use an indirect-gradient approach by computing the gradient of the metamodel, which is often an analytical deterministic function that is also cheaper to evaluate. For a review of methods (2) and (3), see Barton and Meckesheimer (2006). In this paper, we focus on metamodel methods.

Metamodel methods build an analytical and deterministic model that approximates the stochastic outputs (objective function or constraints) based on a sample of simulation observations. By replacing the stochastic simulation response by a deterministic and typically differentiable function, these methods can resort to the use of traditional derivative-based optimization algorithms. Additionally, metamodels are not computationally expensive to evaluate. The main limitation remains the number of simulation runs needed to obtain a good analytical approximation.

Metamodels are classified as either physical or functional metamodels in literature (Søndergaard 2003; Serafini 1998). Functional metamodels are typically general-purpose functions, chosen for their analytical properties. They are often a linear combination of basis functions from a parametric family. The most common choice are low-order polynomials (e.g., linear or quadratic). Other choices include radial-basis functions or spline models. Physical metamodels are application and problem-specific models. Their parameters typically have a physical interpretation. More importantly, their functional form is problem specific.

In past work, we have proposed a metamodel that combines a physical metamodel with a functional metamodel (Osorio and Bierlaire 2010). The functional component is a quadratic polynomial, which ensures asymptotic metamodel properties (needed to analyze asymptotic convergence properties), whereas the physical metamodel provides structural information that enables the method to identify suitable solutions with very small sample sizes. The physical component is an analytical and differentiable queueing network model (Osorio and Bierlaire 2009a; Osorio and Bierlaire 2009b).

Thus, this metamodel combines information from a low-resolution but computationally efficient analytical queueing network model with high-resolution simulated data. This metamodel has allowed us to achieve good short-term performance (Osorio and Bierlaire 2010). In this paper, we propose a scalable formulation of the corresponding metamodel. We then evaluate the performance of this method by addressing a large-scale signal control problem.

This paper is organized as follows. In Section 2, we present our metamodel method. We then present the traffic signal control problem (Section 3). The framework is then applied to address the signal control problem for the entire city of Lausanne in Switzerland (Section 4). Section 5 provides a brief conclusion.

\section{SCALABLE METAMODEL}

The scalable metamodel used in this paper is adapted from Osorio and Bierlaire (2010).

The functional form of the initial metamodel $m$ is: 


\section{Osorio and Chong}

$$
m(x, y ; \alpha, \beta, q)=\alpha T(x, y ; q)+\phi(x ; \beta)
$$

where $\phi$ is the functional component of the metamodel and $T$ is the physical component. The functional component is a quadratic polynomial in the decision vector $x$. The parameters $\alpha$ and $\beta$ are the parameters of the metamodel. The physical component is an analytical approximation of the objective function provided by the queueing model, $y$ are endogenous queueing model variables (e.g., queue-length probabilities) and $q$ are exogenous queueing model parameters (e.g., total demand).

Simulated observations are collected from one iteration to the next. The parameters of the metamodel are fitted based on the simulated outputs by solving a least squares problem. This is done iteratively: as more simulation observations become available, the metamodel is refitted and used to identify points with improved performance. The metamodel is embedded within a derivative-free trust region algorithm proposed by Conn and Scheinberg (2009). For more details, we refer the reader to Osorio and Bierlaire (2010).

The physical model is based on finite capacity queueing network theory (FCQT). It models each lane of an urban road network as a queue. Through a system of nonlinear differentiable equations, the model approximates the between-lane interactions, i.e., it approximates analytically how a lanes state is related to its upstream and downstream lanes. This physical model provides a macroscopic description of the complex spatial interactions congested traffic (e.g., spillback probabilities). The model yields a variety of probabilistic performance measures, such as queue-length probability distributions for each lane. The scalable queueing model used in this paper, is derived from this initial queueing network model.

The following notation is used to formulate the scalable queueing model. The index $i$ refers to a given queue.

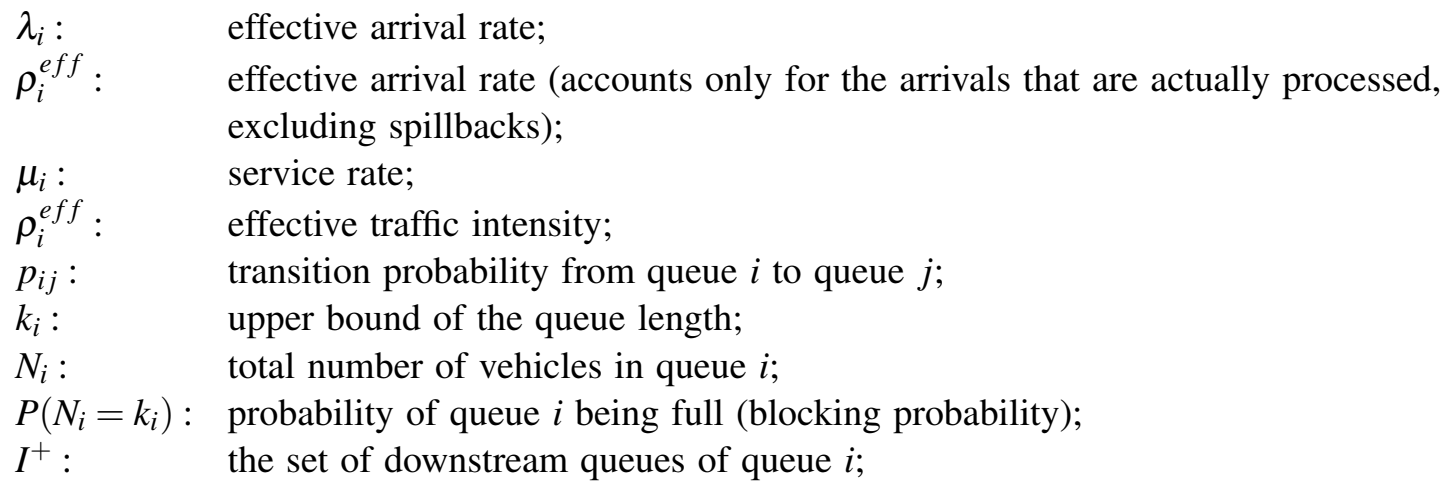

The initial model proposed by Osorio and Bierlaire (2010) is not sufficiently tractable to solve large-scale problems (e.g., city-wide transportation problems). This paper uses a formulation derived from the initial model with enhanced tractability, and evaluates its ability to solve city-wide signal control problems

The scalable queueing network model is given by:

$$
\left\{\begin{array}{l}
\lambda_{i}^{e f f}=\gamma_{i}\left(1-P\left(N_{i}=k_{i}\right)\right)+\sum_{j} p_{j i} \lambda_{i}^{\text {eff }} \\
\rho_{i}^{\text {eff }}=\frac{\lambda_{i}^{\text {eff }}}{\mu_{i}}+\sum_{j \in I^{+}} p_{i j} P\left(N_{j}=k_{j}\right) * \sum_{j \in I^{+}} \rho_{j}^{\text {eff }} \\
P\left(N_{i}=k_{i}\right)=\frac{1-\rho_{i}^{e f f}}{1-\rho_{i}^{e f k_{i}+1}} \rho_{i}^{e f f} k_{i}
\end{array}\right.
$$

The first equation of the System of Equations (2) is a flow conservation equation, relating demand on upstream queues to that on downstream queues. The second equation in the System of Equations (2) associates the traffic intensity of a queue with the parameters of its downstream queues. The third equation 


\section{Osorio and Chong}

yields the probability that a finite capacity queue is full and is derived from finite capacity queueing theory (Bocharov, D'Apice, Pechinkin, and Salerno 2004). For a given queue $i, \gamma_{i}, p_{i j}$ and $k_{i}$ are exogenous parameters. For all queues, the vector of exogenous parameters is denoted as $q$ in the System of Equations (2). Each queue $i$ has three endogenous variables: $\rho_{i}^{\text {eff }}, \lambda_{i}^{\text {eff }}$ and $P\left(N_{i}=k_{i}\right)$.

This formulation considers a set of three endogenous variables $\left(\rho_{i}^{\text {eff }}, \lambda_{i}^{\text {eff }}\right.$ and $P\left(N_{i}=k_{i}\right)$ ) for each queue, whereas the initial formulation considered five. For each queue, its variables are defined through one linear equation, and two nonlinear equations. This novel formulation allows us to reduce the number of endogenous variables. It consists of a simple system of linear and nonlinear equations, that can be efficiently used to solve problems for large-scale networks.

The details of how this formulation is derived from the initial queueing network model can be found in (Osorio and Chong 2012). The main difference between both formulations, is that the more tractable one replaces the traffic intensity of a queue $\rho_{i}$ with the effective traffic intensity $\rho_{i}^{\text {eff }}\left(\rho_{i}^{\text {eff }}=\rho_{i}\left(1-P\left(N_{j}=k_{j}\right)\right)\right.$. For scenarios with low to medium levels of congestion, the two models yield similar estimates. For highly congested scenarios the tractable formulation may underestimate the traffic intensity of the underlying lanes. In this paper, the tractable formulation is used to solve a large-scale signal control problem. We therefore evaluate its ability to identify signal plans that mitigate city-wide congestion levels.

\section{TRAFFIC SIGNAL CONTROL PROBLEM}

\subsection{Signal Control Problem}

In order to evaluate the performance of our method, we consider a traffic signal control problem as formulated in Osorio and Bierlaire (2010). We describe the definitions of the corresponding traffic terminology briefly. More details are included in Osorio and Bierlaire (2009b).

The objective of traffic control problem is to minimize expected travel time by adjusting signal plans at several intersections throughout a city. Cycle length, green splits and offsets are the three main control variables. A phase is defined as a set of streams that are mutually compatible and that receive identical control. Green splits are the normalized durations of phases. Offsets are defined as the differences in time between the beginnings of cycles of adjacent intersections.

We consider a fixed-time control problem where the cycle times, offsets, all-red durations (the length of time when all intersection approaches receive red signal) and phase sequence of intersections are fixed. The green time durations are the only decision variables in our problem. The fixed-time control strategy is a simple signal control technique suitable for congested urban networks. To formulate this problem, we introduce the following notation.
$b_{i}: \quad$ available cycle ratio of intersection $i$ (available green time divided by cycle length);
$x(j): \quad$ green split of phase $j$;
$x_{L}: \quad$ vector of minimal green splits;
$\mathscr{I}: \quad$ set of intersection indices;
$\mathscr{P}_{I}(i)$ : set of phase indices of intersection $i$.

The problem is formulated as follows,

$$
\min _{x} E[f(x, y ; p)]
$$

subject to

$$
\begin{array}{r}
\sum_{j \in \mathscr{P}_{I}(i)} x(j)=b_{i}, \quad \forall i \in \mathscr{I} \\
x \geq x_{L} .
\end{array}
$$




\section{Osorio and Chong}

The green splits of the phases are the decision variables. The decision vector is denoted as $x$. Equation (5) states that the available cycle time at a given intersection should be fully allocated across the different phases. Equation (4) represents lower bounds for the green splits, these have been set according to Swiss transportation standards (VSS 1992).

\subsection{SO Algorithm}

The optimization algorithm used is the same as that in Osorio and Bierlaire (2010). It is based on a derivative-free trust region (TR) algorithm, proposed by Conn and Scheinberg (2009). This framework allows for arbitrary metamodels and makes no assumption on how these metamodels are fitted (interpolation or regression). To ensure global convergence, a model improvement algorithm guarantees that the models achieve a uniform local behavior (i.e., satisfy Taylor-type bounds) within a finite number of steps.

At a given iteration $k$ the trust region subproblem includes more constraints than the previous problem, and is given by:

$$
\min _{x, y} m_{k}=\alpha_{k} T(x, y ; q)+\phi\left(x ; \beta_{k}\right)
$$

subject to

$$
\begin{array}{r}
\sum_{j \in \mathscr{P}_{I}(i)} x(j)=b_{i}, \forall i \in \mathscr{I} \\
h(x, y ; q)=0 \\
\left\|x-x_{k}\right\|_{2} \leq \Delta_{k} \\
y \geq 0 \\
x \geq x_{L}
\end{array}
$$

where $x_{k}$ is the current iterate (i.e., the signal plan that is currently considered to have best performance), $\Delta_{k}$ is the current trust region radius, $\alpha_{k}$ and $\beta_{k}$ are the current metamodel parameters.

Equations (7) and (11) are the signal control constraints, previously described. Equation (8) represents the scalable queueing model formulation, which corresponds to the System of Equations (2). Constraint (9) is the trust region constraint. It uses the Euclidean norm (Conn and Scheinberg 2009). The endogenous variables of the queueing model are subject to positivity constraints (Equation (10)). Thus, the trust region subproblem consists of a nonlinear objective function subject to nonlinear and linear equalities, a nonlinear inequality and bound constraints.

The component $T$ of the objective function in Equation (6) is an approximation of the expected travel time derived based on Little's law and is given by:

$$
T(x, y ; q)=\frac{\sum_{i} E\left[N_{i}\right]}{\sum_{i} \gamma_{i}\left(1-P\left(N_{i}=k_{i}\right)\right.}
$$

where $E\left[N_{i}\right]$ is the expected number of vehicles in queue $i$ and is given by:

$$
E\left[N_{i}\right]=\rho_{i}\left(\frac{1}{1-\rho_{i}}-\left(k_{i}+1\right) \frac{\rho_{i}^{k_{i}}}{1-\rho_{i}^{k_{i}+1}}\right)
$$

In Equations (12) and (13), the traffic intensity $\rho_{i}$ is approximated by the effective traffic intensity $\rho_{i}^{\text {eff }}$. The details of how Equations (12) and (13) are derived are given in Osorio (2010).

For a problem with $l$ lanes (i.e., queues) and $n$ endogenous phases, the problem is implemented with $3 l+n$ endogenous variables, consisting of 3 endogenous queueing model variables per lane, and the green 


\section{Osorio and Chong}

splits for each phase. The trust-region constraint (9) is implemented as an inequality constraint. Lower bounds are implemented as nonlinear equalities via a change of variable. This problem is solved with the Matlab routine for constrained nonlinear problems, fmincon with an interior-point method (Coleman and Li 1996). We set the tolerance for relative change in the objective function to $10^{-3}$ and the tolerance for the maximum constraint violation to $10^{-2}$.

\section{EMPIRICAL ANALYSIS}

We evaluate and illustrate the use of the proposed SO framework with case studies based on road networks for the entire Swiss city of Lausanne. We use a calibrated microscopic traffic simulation model of the Lausanne city center. This model (Dumont and Bert 2006) is implemented with the AIMSUN simulator (TSS 2008).

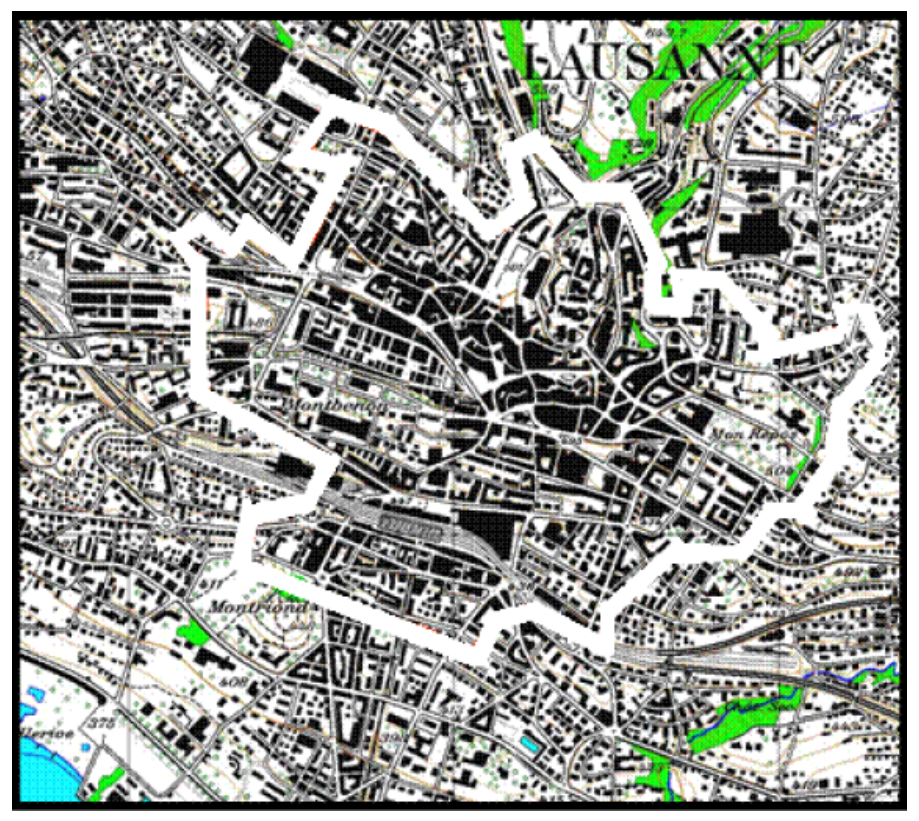

Figure 1: Lausanne city road network (adapted from Dumont and Bert, (2006).)

The Lausanne city road network is displayed in Figure 1. The corresponding network model is given in Figure 2. The model considers 603 roads and 231 intersections. We determine the fixed-time signals of 17 intersections, with cycle time of either 80,90 or 100 seconds. A total of 99 signal phases are endogenous (i.e., the dimension of the decision vector is 99). The 17 controlled intersections are depicted as filled squares in Figure 2. Further, details regarding the Lausanne network are given in Osorio (2010).

The queueing model consists of 902 queues. The optimization problem consists of 2805 endogenous variables with 1821 nonlinear equality constraints and 902 linear equality constraints. This problem is considered of very large-scale for existing unconstrained derivative-free algorithms, not to mention the added complexity of nonlinear constraints and stochasticity.

The considered scenario consists of the evening peak period (17h-18h). The lower bounds of the green splits $\left(x_{L}\right.$ in Equation (11)) are set to 4 seconds according to the Swiss transportation norm (VSS 1992).

We compare the performance of the scalable metamodel with a traditional metamodel method that only uses a quadratic polynomial with diagonal second derivative matrix (in other words, the metamodel consists of $\phi$ ) only). In order to compare the two methods, we consider a tight computational budget, which is defined as a maximum of 150 simulation runs that can be carried out.

We consider three different initial points (i.e., signal plans). These points are uniformly drawn from the feasible space defined by constraints (4) and (5). For each initial point, we run the SO algorithm 


\section{Osorio and Chong}

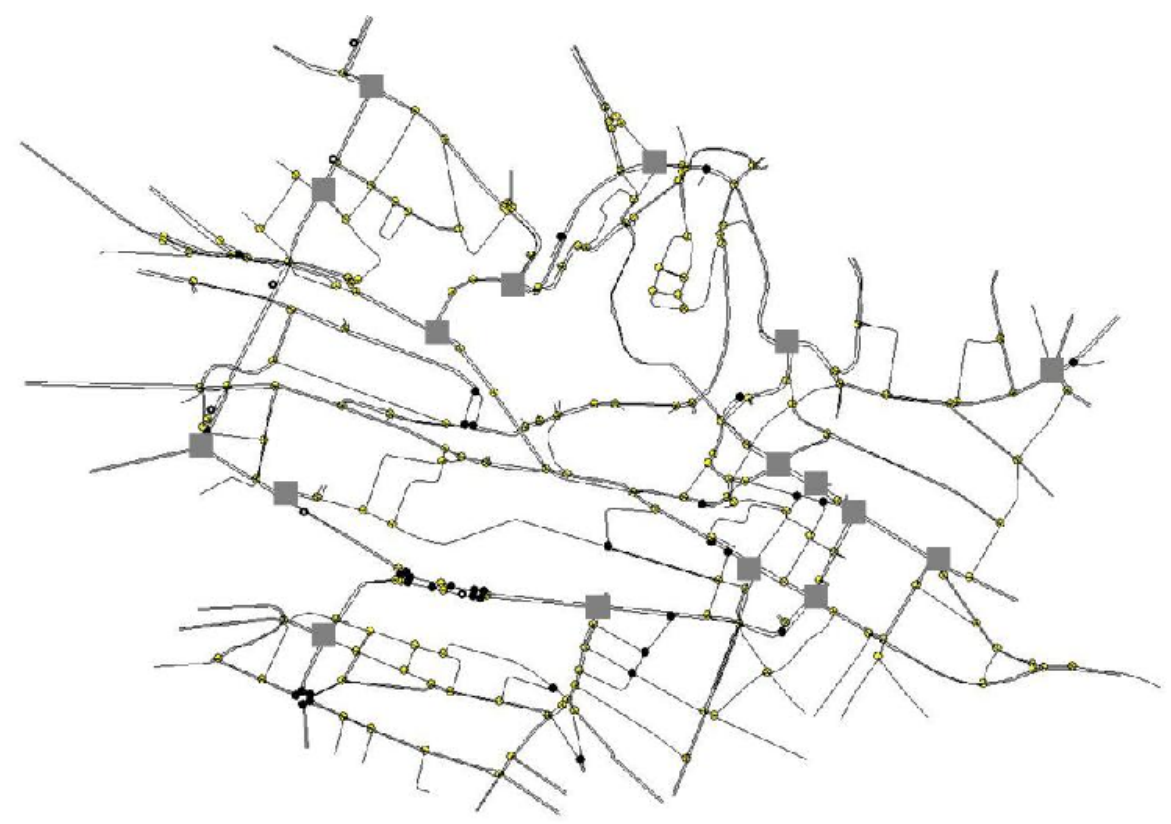

Figure 2: Lausanne network model.

five times. Thus, for each method we derive five "optimal" plans for each initial point. We then use the simulator to evaluate in detail the performance of the derived signal plans. For each derived "optimal" signal plan, we run 50 replications. We then compare the empirical cumulative distribution function (cdf) of the average travel times obtained from these 50 replications.

Figure 3 and Figure 4 display the empirical cdfs of the average travel times for each of the two different initial points. Each curve is a cdf of a given signal plan. The solid thick curve corresponds to the cdf of the initial signal plan, the solid thin (respectively, the dashed) curves are cdf's of the signal plans derived by the proposed (respectively, traditional) approach.

Figure 3 shows that the polynomial derives one signal plan with worse performance than the initial plan, two with similar performance, and two with improved performance. All five signal plans derived by the proposed metamodel yield improved performance when compared to the initial plan. One of the signal plans proposed by the polynomial outperforms all signal plans derived by the proposed metamodel.

In Figure 4, one signal plans proposed by the polynomial metamodel have worse performance than the initial signal plan, two have similar performance and two have improved performance. All five plans derived by the proposed metamodel yield improvement compared to the initial plan, three of them outperform all signal plans proposed by the polynomial.

For all two initial points, the proposed method systematically derives signal plans with improved performance when compared to the initial plan, and most often, when compared to the plans obtained from the polynomial metamodel method.

We now allow for a large computational budget. 600 simulation runs are carried out only once, using the first initial signal plan. In order to evaluate how the performance of the proposed and the traditional metamodel methods change as the sample size increases, we evaluate the performances of the "optimal" plans at sample sizes 50,150, 200, 400, and 600. In order to evaluate the performance of the signal plans, we proceed as above (i.e., we run 50 replications and compare the cdf's of the average travel times).

Figure 5 shows the performance of the derived "optimal" plans at different sample sizes. The plan derived by the proposed approach (solid thin cdf curve) at sample size 50 is the same as that at sample size 600. It has improved performance compared to the initial signal plan (thick solid cdf). The dashed cdf's correspond to signal plans derived by the traditional polynomial metamodel approach. They correspond 


\section{Osorio and Chong}

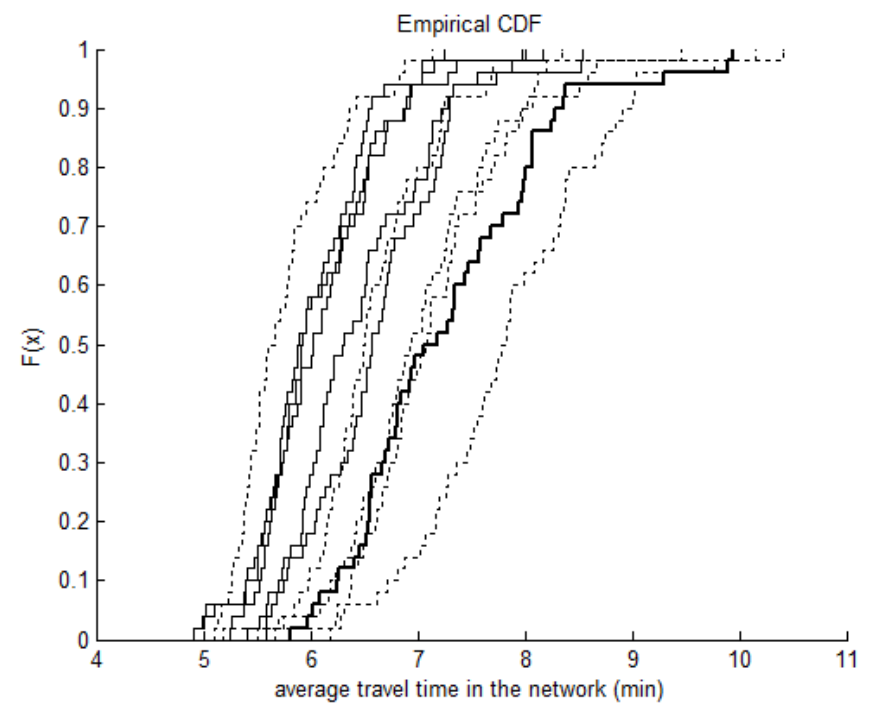

Figure 3: Empirical cdfs of the average travel times in the full city network using a given initial point 1. Solid thick: initial plan, Solid thin: proposed metamodel, Dashed: polynomial.

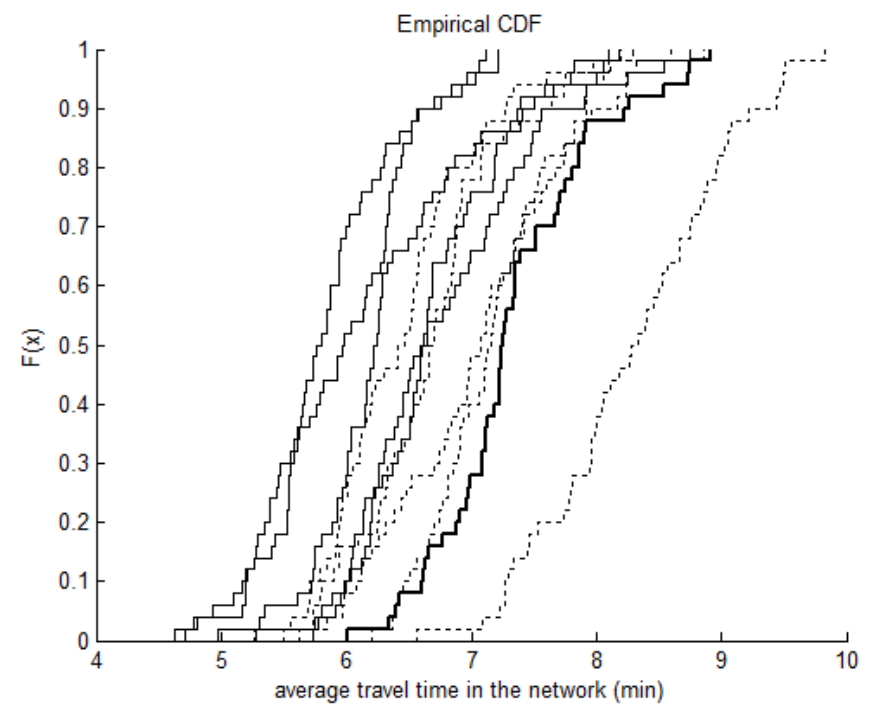

Figure 4: Empirical cdfs of the average travel times in the full city network using initial point 2. Solid thick: initial plan, Solid thin: proposed metamodel, Dashed: polynomial. 


\section{Osorio and Chong}

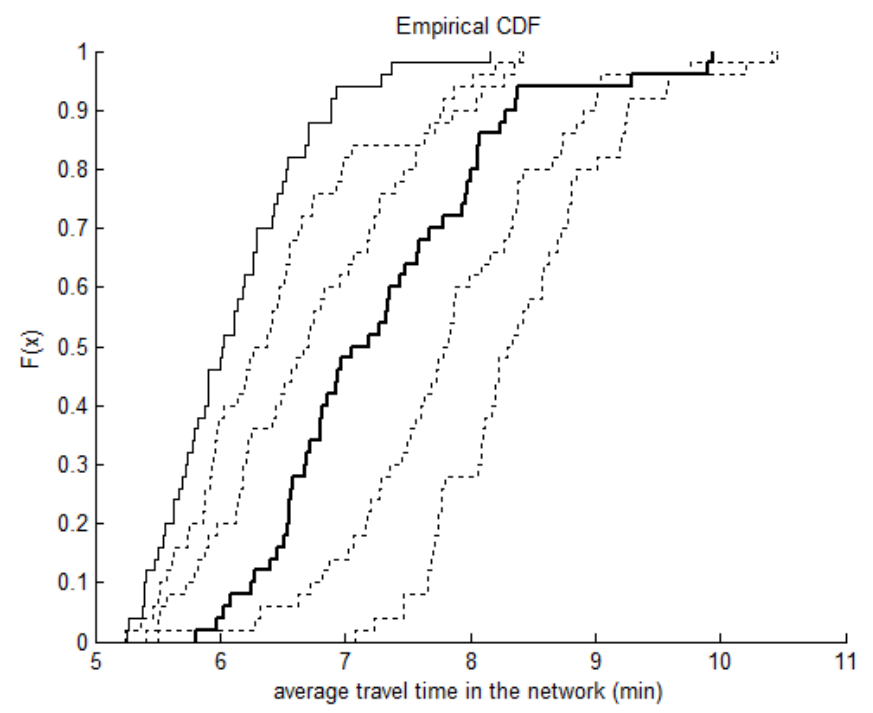

Figure 5: Empirical cdfs of the average travel times in the full network using initial point 1 with large sample size.

from right to left to sample sizes 50, 150/200, 400, 600. The "optimal" plan at sample size 150 is the same as that at size 200. As the sample size increases the traditional method identifies signal plans with improved performance. Their performance at sample size 600 is still inferior to that of the proposed method.

\section{CONCLUSION}

This paper applies a simulation-based optimization algorithm suitable to address large-scale problems under tight computational budgets. It uses a metamodel that combines a general-purpose component (a quadratic polynomial) with a physical component which is a scalable analytical queueing network model. We evaluate the performance of this approach by addressing a large-scale signal control problem for the entire city of Lausanne, Switzerland. We compare the performance of the scalable metamodel to that of a traditional metamodel. Our method identifies signal plans that improve the distribution of travel times compared to both the initial signal plans, and most often, to the signal plans derived by the traditional metamodel method.

This approach allows us to formulate and solve a variety of challenging large-scale transportation optimization problems that mitigate congestion while enhancing the sustainability of the transportation network. For instance, we have recently addressed an energy-efficient signal control problem (Osorio and Nanduri 2012), where the traffic simulator is coupled with detailed vehicle-specific instantaneous (also known as microscopic) fuel consumption simulators. The integrated models are then used to derive signal plans that reduce both travel times and fuel consumption.

As part of this ongoing research, we are currently further enhancing the scalability of this approach. We are also developing SO algorithms with improved short-term performance by using analytical low-resolution (e.g., macroscopic) probabilistic models, such as the queueing model used in this paper, to inform both sampling strategies and statistical testing. 


\section{Osorio and Chong}

\section{REFERENCES}

Barton, R. R., and M. Meckesheimer. 2006. "Metamodel-based simulation optimization". In Handbooks in operations research and management science: Simulation, edited by S. G. Henderson and B. L. Nelson, Volume 13, Chapter 18, 535-574. Amsterdam: Elsevier.

Bocharov, P. P., C. D'Apice, A. V. Pechinkin, and S. Salerno. 2004. "Queueing theory". In Modern Probability and Statistics, Chapter 3, 96-98. Zeist, Te Neitherlands: Bill Academic Publishers.

Chen, X., C. Osorio, and B. F. Santos. 2012. "(Submitted). A simulation-based approach to reliable signal control". International Symposium on Transportation Network Reliability (INSTR).

Coleman, T. F., and Y. Li. 1996. "An Interior, Trust Region Approach for Nonlinear Minimization Subject to Bounds". SIAM Journal on Optimization 6:418-445.

Conn, A. R., and K. Scheinberg. 2009. "Introduction to derivative-free optimization". MPS/SIAM Series on Optimization, Society for Industrial and Applied Mathematics and Math-ematical Programming Society.

Dumont, A. G., and E. Bert. 2006, May. "Simulation de l'agglomération Lausannoise SIMLO”. Technical report, Laboratoire des voies de circulation, ENAC, Ecole Polytechnique Fédérale de Lausanne.

Kolda, T., R. M. Lewis, and V. Torczon. 2003. "Optimization by Direct Search: New Perspectives on Some Classical and Modern Methods". SIAM Review 45 (3): 385-482.

Osorio, C. 2010. Mitigating network congestion: analytical models, optimization methods and their applications. Ph. D. thesis, Ecole Polytechnique Federale de Lausanne, Lausanne, Switzerland.

Osorio, C., and H. Bidkhori. 2012, December. "Combining metamodel techniques and Bayesian selection procedures to derive computationally efficient simulation-based optimization algorithms". In Proceedings of the 2012 Winter Simulation Conference, edited by C. Laroque, J. Himmelspach, R. Pasupathy, O. Rose, and A. M. Uhrmacher. Piscataway, New Jersey: Institute of Electrical and Electronics Engineers, Inc.

Osorio, C., and M. Bierlaire. 2009a. "An analytic finite capacity queueing network model capturing the propagation of congestion and blocking". European Journal of Operational Research 196 (3): 996-1007.

Osorio, C., and M. Bierlaire. 2009b, August. "A surrogate model for traffic optimization of congested networks: an analytic queueing network approach". Technical Report 090825, Transport and Mobility Laboratory, ENAC, Ecole Polytechnique Fédérale de Lausanne.

Osorio, C., and M. Bierlaire. 2010, June 20-25. "A simulation-based optimization approach to perform urban traffic control". In Proceedings of the Triennial Symposium on Transportation Analysis (TRISTAN). Troms $\varnothing$, Norway.

Osorio, C., and L. Chong. 2012. "Large-scale simulation-based traffic signal control". In International Symposium on Dynamic Traffic Assignment (DTA). Marthas Vineyard, USA.

Osorio, C., and K. Nanduri. 2012. "Energy-efficient traffic management: a microscopic simulation-based approach". In International Symposium on Dynamic Traffic Assignment (DTA). Marthas Vineyard, USA.

Serafini, D. B. 1998. A framework for managing models in nonlinear optimization of computationally expensive functions. Ph. D. thesis, Rice University.

Søndergaard, J. 2003. Optimization using surrogate models - by the Space Mapping technique. Ph. D. thesis, Technical University of Denmark.

TSS 2008, May. AIMSUN NG and AIMSUN Micro Version 5.1. Transport Simulation Systems.

VSS 1992. Norme Suisse SN 640837 Installations de feux de circulation; temps transitoires et temps minimaux. Zurich: Union des professionnels suisses de la route.

\section{AUTHOR BIOGRAPHIES}

CAROLINA OSORIO is an Assistant Professor of Civil and Environmental Engineering (CEE) at the Massachusetts Institute of Technology (MIT). She received her Ph.D from EPFL in 2010. She develops operations research techniques to inform the design and operations of transportation systems. She is inter- 


\section{Osorio and Chong}

ested in techniques that combine ideas from the fields of probability theory, simulation, simulation-based optimization, derivative-free optimization, nonlinear optimization, statistics, traffic control and traffic flow theory. Her email address is osorioc@mit.edu.

LINSEN CHONG is a Ph.D. student in the Department of Civil and Environmental Engineering (CEE) at the Massachusetts Institute of Technology (MIT). He received his masters degree from Virginia Polytechnic Institute and State University in August, 2011. His current research interests include applications of optimization methods especially to traffic management and control. His email address is linsenc@ mit.edu. 